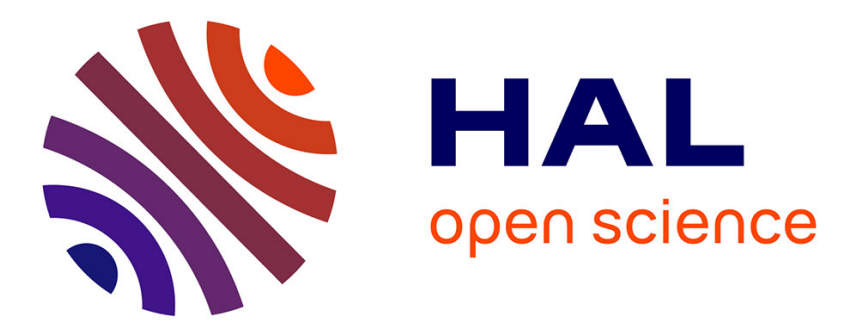

\title{
Why do liver transplant patients so often become obese? The addiction transfer hypothesis
}

\author{
Paul Brunault, Ephrem Salamé, Nematollah Jaafari, Robert Courtois, \\ Christian Réveillère, Christine Silvain, Amine Benyamina, Lisa Blecha, David \\ Belin, Nicolas Ballon
}

\section{To cite this version:}

Paul Brunault, Ephrem Salamé, Nematollah Jaafari, Robert Courtois, Christian Réveillère, et al.. Why do liver transplant patients so often become obese? The addiction transfer hypothesis. Medical Hypotheses, 2015, 85 (1), pp.68-75. 10.1016/j.mehy.2015.03.026 . hal-01379612

\section{HAL Id: hal-01379612 https://hal.science/hal-01379612}

Submitted on 11 Oct 2016

HAL is a multi-disciplinary open access archive for the deposit and dissemination of scientific research documents, whether they are published or not. The documents may come from teaching and research institutions in France or abroad, or from public or private research centers.
L'archive ouverte pluridisciplinaire HAL, est destinée au dépôt et à la diffusion de documents scientifiques de niveau recherche, publiés ou non, émanant des établissements d'enseignement et de recherche français ou étrangers, des laboratoires publics ou privés. 
Title of article: Why do liver transplant patients so often become obese? The addiction transfer hypothesis

Authors:Paul Brunault (MD; Psychiatrist and Addictologist) ${ }^{1,2,3}$, Ephrem Salamé (MD, PhD; Professor of Medicine - Surgery) $)^{4,5,6}$, Nematollah Jaafari (MD; Professor of Medicine - Psychiatry) ${ }^{7}$, Robert Courtois (MD, $\mathrm{PhD}$; Psychiatrist and Associate Professor of Psychology) $)^{2,3}$, Christian Réveillère (PhD; Professor of Psychology) ${ }^{3}$, Christine Silvain (MD; Professor of Medicine - Gastro-enterology \& Hepatology) ${ }^{8}$, Amine Benyamina (MD, PhD; Professor of Medicine - Psychiatry) ${ }^{9}$, Lisa Blecha (MD; Addictologist) ${ }^{9}$, David Belin $(\mathrm{PhD} \text {; Lecturer in Neuroscience })^{10} *$, Nicolas Ballon (MD, PhD; Professor of Medicine - Psychiatry $)^{1,2,11} *$

Names and addresses of the department or institution to which the work should be attributed:

${ }^{1}$ CHRU de Tours, Équipe de Liaison et de Soins en Addictologie, Tours, France

${ }^{2}$ CHRU de Tours, Clinique Psychiatrique Universitaire, Tours, France

${ }^{3}$ Université François Rabelais de Tours, PRES Centre-Val de Loire, Département de Psychologie, EA 2114

«Psychologie des Âges de la Vie », Tours, France

${ }^{4}$ CHRU de Tours, Service de Chirurgie Digestive Endocrinienne et Transplantation Hépatique, Tours, France

${ }^{5}$ Université François Rabelais de Tours, Tours, France

${ }^{6}$ INSERM U1082, Poitiers, France

${ }^{7}$ Centre Hospitalier Henri Laborit, Service Universitaire de Psychiatrie, Poitiers, France

${ }^{8}$ CHU de Poitiers, Service d'Hépato-gastroentérologie, Poitiers, France

${ }^{9}$ Hôpital Universitaire Paul Brousse, Centre d'Enseignement, de Recherche et de Traitement des Addictions, Villejuif, France

${ }^{10}$ University of Cambridge, Department of Pharmacology, Tennis Court Road CB2 1PD Cambridge

${ }^{11}$ UMR INSERM U930 \& CNRS ERL 3106, Tours, France

*: these authors contributed equally to this work

Author responsible for correspondence and to whom requests for offprints should be sent:

Name: Paul Brunault

Address: CHRU de Tours, Équipe de Liaison et de Soins en Addictologie, 2 boulevard Tonnellé, 37000 Tours, France

Telephone number: $+33-247-478043$

Fax number: +33-247-478402

e-mail address: paul.brunault@univ-tours.fr

Sources of support: this study was conducted without specific funding. DB is supported by the University of Cambridge and the Agence Nationale de la Recherche (ANR) grant ANR12 SAMA00201.

This is the postprint version (final draft post refereeing) of the following article: "Paul Brunault, Ephrem Salamé, Nematollah Jaafari, Robert Courtois, Christian Réveillère, Christine Silvain, Amine Benyamina, Lisa Blecha, David Belin*, Nicolas Ballon*. Why do liver transplant patients so often become obese? The addiction transfer hypothesis Medical Hypotheses Jul;85(1):68-75. doi: 10.1016/j.mehy.2015.03.026", which has been published in final form at: http://www.sciencedlirect.com/science/article/pii/S0306987715001413 


\begin{abstract}
:
In patients who receive transplantation for alcohol liver disease, obesity and metabolic syndrome are highly prevalent after transplantation and both contribute to a significant proportion of cardiovascular complications, late morbidity and mortality in this population. Although immunosuppressive medications have been hypothesised to explain some of these post-liver-transplantation (LT) metabolic complications, they cannot be considered the sole cause of obesity and metabolic syndrome, and the high prevalence of these illnesses remains unexplained. Given the significant overlap between the neurobiological, psychiatric and psychological factors that underlie alcohol addiction and reward-related behavioural dyscontrol disorders such as food addiction (FA), we hypothesised that the high prevalence of obesity and metabolic syndrome reported in patients who receive transplantation for alcohol liver disease could be explained at least partially by a switch in some individuals from a previous alcohol addiction to post-transplantation FA (i.e., addiction transfer $=$ addiction switch). In our integrative model, we also speculate that an increased prevalence of FA or alcohol addiction may occur in patients with both specific psychobiological profiles and shared risk factors. We further hypothesise that in the subpopulation of patients who develop either alcohol addiction or FA after LT, those with high insight with regard to the consequences of alcohol use could be at higher risk for FA, whereas those with low insight could be at higher risk for alcohol addiction. We discuss here evidence for and against this hypothesis and discuss which patients could be more vulnerable to these two addictions after LT. Because it will not be either possible or ethical to test some of our hypotheses in humans, future studies should test these hypotheses using a translational strategy, using both clinical and preclinical approaches. If our hypotheses could account for the significant increase in obesity and metabolic syndrome after LT, this would lead to new avenues for research and preventive as well as therapeutic interventions for alcohol-related LT patients. All patients with previous or current alcohol addiction should be systematically screened for FA and followed up for subsequent risk of obesity and metabolic syndrome. Such strategies might be effective in improving survival, outcomes and quality of life after LT and also in the overall population of patients with alcohol addiction. By determining common risk factors for both alcohol addiction and FA using a translational approach, our model could help to find novel psychopharmacological and psychological strategies that might be effective in both FA and alcohol addiction.
\end{abstract}

Keywords:liver transplantation; liver disease; food addiction; obesity; binge eating disorder; compulsive eating behaviour; alcohol dependence; alcohol addiction; alcohol abuse; alcohol use disorder; alcoholism; addiction transfer; switch; insight; psychiatric disorders. 


\section{INTRODUCTION}

Alcohol use disorders are defined by a cluster of cognitive, behavioural, and physiological symptoms that indicate persistent use of a substance despite significant substance-related problems [1]. These disorders are a major public health problem worldwide, with a lifetime prevalence rate in the United States estimated at $17.8 \%$ $(0.5)$ and $12.5 \%$ (0.4) for alcohol abuse and dependence [2], thereby representing a health, social and economical burden to western societies [3]. Approximately 3.8\% of all global deaths and $4.6 \%$ of global disability-adjusted life-years are attributable to alcohol [3]. In France, in 2009, 36,500 deaths in men were attributable to alcohol ( $13 \%$ of total mortality) versus 12,500 in women (5\% of total mortality) [4]. One of the somatic consequences of protracted alcohol addiction is liver cirrhosis or liver failure, for which liver transplantation (LT) has progressively become the best, and sometimes the only therapeutic alternative for patients with end-stage liver failure [5].

However, the success rate of LT in patients suffering from alcohol addiction remains relatively poor. It is critical to better identify the factors that contribute to poorer post-transplant outcomes in former alcohol addicts not only because alcohol use disorders are currently the leading cause for LT [6] but also because these factors lead, when untreated, to lower quality of life and lower survival rates [7]. This is a major public health issue not only for these patients but also because of the economic and social costs associated with liver transplantation and the limited number of organ donors, which prevents other patients with potentially better outcomes from benefiting from this treatment [5].

Obesity and metabolic syndrome are among the main factors that contribute to a significant part of cardiovascular complications and late morbidity and mortality after LT [8]. Some factors such as use of immunosuppressive medications, donor characteristics (e.g., body mass index), and pre-transplant nutritional and metabolic status have been hypothesised to account for some of these post-LT metabolic complications (see for review [9]). However, these hypotheses cannot explain why and how patients with a pre-transplant alcohol use disorder are at higher risk than other liver-transplanted patients for developing post-transplant metabolic syndrome [8,10] and obesity [11]. According to a study conducted by Anastácio et al. in 148 liver transplanted patients with a mean follow-up of 3.5 years [10], prevalence for metabolic syndrome was $84 \%$ higher in patient transplanted for an alcohol liver disease when compared to patients transplanted for a non-alcohol liver disease (prevalence rate for metabolic syndrome were respectively $71.7 \%$ and $38.9 \%$; OR=3.88; 95\% CI=1.43-10.51; $\mathrm{p}<0.001)$.

The elevated rate of metabolic syndrome observed in patients with alcohol use disorder after LT is all the more surprising given that the risk of relapsing to alcohol use after LT, a classic risk factor for post-LT metabolic complications, is lower in these patients than in control populations that are treated for alcohol abuse [12]. During the five years following LT, one out of four and one out of eight patients, respectively, will relapse to any alcohol use or to heavy alcohol use [6], whereas in primary care, more than four patients out of five will relapse to alcohol use in any given 18-month period [13]. We currently don't know whether it is the amount of alcohol intake an/or the duration of alcohol intake that are associated with higher risk for post-transplant metabolic complications, and we alsolack an understanding of why patients who receive transplantation for alcohol liver disease show both an increased risk of metabolic syndrome and obesity and a lower risk of relapse to alcohol addiction after LT.

Although excessive and compulsive food intake may be a prominent contributor to the high incidence of overweight and obesity after LT and in patients with a preoperative alcohol use disorder, data on food intake in patients who receive transplantation for an alcohol use disorder are very scarce [9]. Only Correia et al. mentioned that "non-healthy habits' might be a risk factor for post-transplant obesity [14], but no studies have so far explicitly assessed whether an increased propensity to develop food addiction could be a potential risk factor for later obesity or metabolic syndrome in patients who receive transplantation for an alcohol use disorder. Food addiction, a concept that has been recently proposed by applying the DSM-IV-TR criteria for substance dependence to eating behaviour in order to describe patients who exhibit eating patterns that are similar to the behaviours classically seen in drug-addicted patients [15-17], is indeed strongly associated with obesity [18,19], and it has been hypothesised to play a major role in the recent increase in obesity prevalence in the overall population [20]. 
Finally, it is noteworthy that studies in the field of bariatric surgery have demonstrated that when previous eating behaviour was restricted by bariatric surgery, alcohol use disorders increased [21].

\section{HYPOTHESIS}

Our main hypothesis is that the high rates of obesity and metabolic syndrome reported in post-LT patients could be attributable to a switch from a previous alcohol addiction to a post-LT food addiction (i.e., addiction transfer $=$ addiction switch) in a subpopulation of vulnerable individuals who share certain specific psychobiological risk factors and who also share coping strategies under the peer and moral pressure associated with LT. More specifically, we hypothesise that low insight with regard to the somatic and psychological consequences of alcohol addiction could predict higher risk of alcohol relapse after LT whereas high insight could predict higher risk of developing a food addiction after LT.

We will present a correlational model in which food addiction could partially account for the variance in obesity and metabolic syndrome observed post LT in former alcohol addicts, in addition to other factors such as pre-transplant nutritional status, use of medications and donor's nutritional status.

\section{HYPOTHESIS EVALUATION}

Our hypothesis is based on four different assumptions regarding the population of patients who receive transplantation for alcohol liver disease:

1) The prevalence of alcohol addiction decreases, while the prevalence of food addiction increases, after LT.

2) A significant part of the weight gain, obesity and metabolic syndrome after LT is attributable to the increased prevalence of food addiction after $L T$.

3) The increased prevalence of food addiction or alcohol relapse after LT occurs in patients with specific psychobiological risk factors.

4) In patients who display addictive behaviours post-LT, the level of insight with regard to the somatic and psychological consequences of alcohol use before LT predicts either the development of a food addiction or the resuming of their former alcohol addiction after LT.

We will review here the pros and cons in support of each of these assumptions before addressing the added value of our hypothesis to the theoretical framework of post-LT complications and potentially refined treatment strategies. We will also describe the potential operationalisations that will be necessary to test our hypothesis.

1) In the specific population of patients transplanted for alcohol-induced liver disease, the prevalence of alcohol addiction decreases, while the prevalence of food addiction increases, after LT.

To explain the fact that the risk of relapsing to alcohol use after LT is lower than it is in control populations that are treated for alcohol abuse [12], a number of hypotheses might be proposed. First, these patients do face a lifethreatening illness, and they receive better social support through improved medical follow-up. Additionally, and perhaps even more importantly, patients benefit from the sustained support of peers and health professionals. Both factors may interact to greatly contribute to subsequently diminishing the risk of alcohol relapse through improved motivation to stop alcohol use, improved perceived social support and improved insight with regard to the somatic and psychological consequences of alcohol addiction. Third, alcohol use after LT might be restricted by heavy social pressure because LT is a rare and costly procedure. Considering this hypothesis, former alcohol addicts who crave alcohol after LT might cope by switching to a more socially accepted or less stigmatised substance or behaviour that could produce similar rewarding effects, thereby increasing the risk for a potential addiction to another reinforcer.

In that context, highly palatable food might be a reinforcerof choice because it is widely available and not yet socially stigmatised or associated with liver cirrhosis in the way that alcohol or illicit drugs are, and it has similar neurobiological properties to alcohol and other drugs [17,22-24]. Both drug use and the intake of certain 
foods (e.g., sugar) have been associated with alterations to the brain's reward systems, including dopamineergic, opioidergic, cholinergic, serotoninergic, and GABAergic systems [20,25], an observation that, along with other neuro-imaging studies, has yielded to the concept of overlapping processes between food and drug cravings [22]. Multiple lines of research have also demonstrated that food-addicted and drug-addicted individuals share common alterations in brain circuitry, including impaired dopaminergic pathways [26-28] such as those that regulate the neuronal systems associated not only with reward sensitivity and incentive motivation but also with conditioning (memory/learning), impulse control (behavioural inhibition), stress reactivity, and interoceptive awareness [28]. More specifically, both ventral striatal (predominantly associated with reward and motivation) and dorsal striatal (associated with habits or stimulus response learning) networks were found to be involved in both drug addiction and food addiction [28]. Recent studies have reported similar patterns of neural activation in addiction-like eating behaviours and substance dependence [23,29]. Studies have found elevated activation of the reward circuitry (the amygdala, anterior cingulate cortex, medial orbitofrontal cortex, dorsolateral prefrontal cortex and caudate) in response to food-related cues associated with the reduced activation of inhibitory regions (lateral orbitofrontal cortex) in response to food intake [23]; these regions are the same neural substrates that are hypothesized to underlie drug addiction [23,29]. These results have been confirmed in animal studies, in which the consumption of sugar-rich foods or drinks is associated with the release of endorphins and dopamine within the nucleus accumbens, akin to some drugs of abuse such as alcohol [30]. Not only does exposure to highly palatable food trigger neurobiological responses that are, at least qualitatively, similar to those of addictive drugs but both preclinical and clinical research has demonstrated that sugary and salty foods both induce withdrawal [17], which, at least for sugar, is associated with an imbalance in the acetylcholine and dopamine systems in the same manner as has been shown for opiate withdrawal [22].

Our hypothesis of an increased risk of food addiction in former alcohol addicts also stems from the clinical observation that former drug addicts who maintain abstinence display an increased preference for sweetness and tend to develop sugar addiction [30,31]. Former drug addicts also report significant weight gain during abstinence $[31,32]$. Some other studies conducted in patients treated for a substance use disorder found that abstinence from alcohol use was associated with significant weight gain, increase in BMI [33] and incidence of bulimia [34], suggesting higher food intake and potentially higher risk for food addiction after alcohol abstinence. In addition, when previous eating behaviour was restricted by bariatric surgery in patients who were obese - which is highly comorbid with food addiction [19] - alcohol use disorders increased [21]. Because this latter study suggests that a transfer from preoperative food addiction to postoperative alcohol addiction is possible, we can also assume that a transfer is also possible from preoperative alcohol addiction to postoperative food addiction in a subpopulation of vulnerable individuals.

Altogether, these data suggest close inter-relationships between food and alcohol addiction in that a decrease in one addiction is frequently associated with an increase in the other. However, the mechanisms by which the switch from one to the other occurs and the potential risk factors that might account for the switch remain to be established. Future studies should also take into account potential confounding factors to determine that these two observations are causally related.

\section{2) A significant part of the weight gain, obesity and metabolic syndrome after LT is attributable to the increased prevalence of food addiction after $L T$.}

We also hypothesise that significant proportions of the weight gain, obesity and metabolic syndrome that follow LT could be causally explained by the increased prevalence of food addiction after LT. Although no longitudinal studies have demonstrated that food addiction directly causes weight gain and obesity, perhaps because the clinical definition of food addiction is very recent, cross-sectional studies have demonstrated that food addiction is highly prevalent among obese patients [18,19]. Similarly, loss of control over eating - which is a key symptom of food addiction - predicted subsequent excessive weight gain among children and adolescents [35]. There is also some evidence that loss of control over eating in childhood is associated with both the subsequent development of components of metabolic syndrome and later overweight and obesity [35,36].

Because obesity is a complex, multifactorial disease [37], other factors in addition to preoperative alcohol addiction could explain part of its increased prevalence following LT in former alcohol addicts. In liver- 
transplanted patients, intake of specific foods might also be facilitated by some of the medications that are frequently used after LT, such as steroids, which contribute to weight gain either through enhanced appetite (direct effect on food intake) or by stimulating the intake of sweet and high-fat foods (which could have an indirect effect on food intake through compulsive eating behaviour) [9]. Tobacco withdrawal is another factor that has often been associated with weight gain [38], but no studies have investigated this hypothesis in the specific population of liver-transplanted patients.

To explain the higher risk of obesity and metabolic syndrome after LT in former alcohol addicts, the impact of altering some metabolic functions consecutive to alcohol consumption could also be considered. Continuous or transient effects of alcohol on the autonomic nervous system and on the transport and metabolism of glucose might indeed be related to an increased concentration of glucose and triglycerides and high blood pressure, leading to higher risk of metabolic syndrome [39]. Leptin, a hormone implicated in regulating appetite, body weight and energy homeostasis, was found to be elevated in alcohol-dependent patients, and LT-associated alcohol abstinence may alter the function of this peptide thereby influencing the development of metabolic syndrome [39]. Although none of these factors alone can explain the higher risk of metabolic syndrome in this population, future studies should assess all of these possible risk factors for obesity in addition to those mentioned in our food addiction hypothesis.

3) The increased prevalence of food addiction or alcohol relapse after LT occurs in patients with specific psychobiological risk factors.

To explain the increased risk of these addictions, we will first discuss the risk factors that are typically shared among a wide range of addictions, including alcohol and food addiction, and then the factors that are more specific to a given addiction. Among these shared risk factors, some psychobiological factors might be associated with both food and alcohol addiction given that overlaps between binge eating and binge drinking have been demonstrated. These risk factors might include certain executive dysfunctions that are shared between binge eating and binge drinking, such as impaired cognitive flexibility or shifting attention [40,41] and some psychiatric comorbidities such as mood disorders, anxiety disorders [42,43], and attention deficit hyperactivity disorder $[44,45]$. Shared risk factors also include some stable personality features such as high neuroticism, low agreeableness, and low conscientiousness [46] (as well as high impulsivity [46,47]) that binge eating and binge drinking also share. We can thus assume that having such a personality and/or psychiatric profile would lead to a higher risk of post-transplant food/alcohol addiction. To our knowledge, no studies to date have assessed these dimensions in an integrative framework as potential risk factors for alcohol or food addiction in livertransplanted patients as well as in the overall population. Among the potential genetic risk factors, variants of the D2 dopamine receptor gene could be considered following the fact that Blum et al. hypothesised that the substitution of food addiction for other dependencies such as alcohol might be attributable to a specific reward deficiency syndrome (indeed, a core component of addiction) that has common genetic determinants such as variants of the D2 dopamine receptor gene, especially the DRD2 A1 allele [22].

Although food addiction and alcohol addiction do share some common risk factors, different risk factors might also be implicated specifically in the development of either food or alcohol addiction. Not all patients who abstain from alcohol use will eventually develop food addiction, and we might also assume that some factors may play a protective role against one addiction or the other. As demonstrated by Volkow, although eating craved foods and drug addiction both activate the reward circuitry that involves dopaminergic pathways, these actions activate these pathways in different ways [48]. Food addiction affects the reward circuitry through endogenous opioids and cannabinoids, whereas drugs affect the same circuitry through direct effects on dopaminergic neurons or indirectly via other neurotransmitters [49]. In addition, the neurobiological regulation of feeding is much more complex than the regulation of drug intake because food consumption is controlled not only by reward but also by multiple peripheral, endocrine and central factors beyond those that participate in reward [48]. In line with this hypothesis, current studies indicate more widespread abnormalities in obesity and eating disorders than in drug addictions.

One interesting hypothesis comes from animal research, which has demonstrated that orexins are involved in motivation for drug use as well as natural rewards [50-53], providing an interesting theoretical framework whereby the underpinnings of compulsive alcohol use may facilitate the emergence of compulsive 
food intake in previously alcohol-dependent patients after LT. Indeed, administration of orexins has been shown to stimulate food consumption [54]. In self-administration studies, the orexin 1 receptor antagonist SB-334867 attenuated instrumental responses for high-fat pellets, sucrose pellets and ethanol but not cocaine, demonstrating that signalling at orexin receptors is necessary for mediating the reinforcing properties of specific rewards [51]. If altering the orexinergic system and orexin-dependent plasticity processes that sub-serve alcohol intake may facilitate the development of aberrant food intake under abstinence [51], further investigation is warranted to determine whether the function or dysfunction of the orexin system may contribute to the overeating associated with obesity in former alcoholics with LT. Similarly, alcohol-associated recruitment of the stress system in rats, including the activation of corticotropin releasing factor receptor 1 (CRF-R1) within the cortico-striatal circuitry may provide a long-lasting substrate to individual vulnerability to relapse to compulsive behaviours after stress $[55,56]$.

As another example of a potential difference in terms of risk factors, alterations in the medial temporal and superior frontal regions that are functionally connected with the dorsal striatum could also be studied further given that they are associated with a greater vulnerability to obesity and eating disorders than to drug addictions [57]. Future studies should assess factors that could play either a protective or a vulnerability role in influencing relapse to either alcohol intake or food addiction.

4) In patients who display addictive behaviours post-LT, the level of insight with regard to the somatic and psychological consequences of alcohol use before LT predicts either the development of a food addiction or the resuming of their former alcohol addiction after $L T$

In addition to the risk factors that are either common to both food and alcohol addiction or specific to a given addiction, the dimension of insight should also be considered. In patients who do either relapse to alcohol use or develop food addiction after LT, we hypothesise here that an increased prevalence of alcohol addiction after LT occurs more frequently in a specific subpopulation that is characterised by little insight into the cause of the liver failure, namely, alcohol addiction (see Figure 1).

The concept of insight refers here to the ability to recognise one's own mental illness, with its causes and consequences [58], and refers to patients' awareness and understanding of their attitudes, feelings, behaviours, and symptoms. Insight encompasses two different dimensions: symptom insight, the patient's awareness of his symptoms, and attribution, which refers to how the patient causally attributes his illness [59]. Because lower insight into alcohol addiction has been associated with poorer outcomes following treatment [60], we assume that in patients who develop either food or alcohol addiction after LT, those who attribute their liver disease to a cause other than alcohol (low attribution) are at higher risk of relapsing to alcohol addiction than are patients with non-impaired insight (Figure 1). More specifically, patients who receive transplantation for alcohol liver disease may be at higher risk than other patients of developing a post-transplant food addiction because they may, under the peer and moral pressure associated with LT, manage their addiction by displacing the object of their craving from alcohol to food, the latter being considered a non-dangerous source of reward relative to the former (i.e., addiction transfer). We suggest here that insight with regard to the somatic and psychological consequences of alcohol should be addressed specifically in addition to the other well-known post-LT risk factors for alcohol relapse that we have previously discussed.

\section{HOW SHOULD WE TEST OUR HYPOTHESES?}

The best approach to testing this model appears to be one that relies on a translational design. Indeed, in addition to the clinical usefulness of human models, animal models allow for a direct causal understanding of the neurobiological and pharmacological factors that cannot be assessed in humans for obvious ethical reasons. Additionally, preclinical studies allow for a complete longitudinal approach that gives insights into the contribution of premorbid risk factors to the switch from alcohol addiction to food addiction, as illustrated in the addiction field [61,62].

In human studies, we will first investigate, within a longitudinal study, both pre- and post-LT alcohol addiction and food addiction over a period of at least three years to screen for a sufficient number of patients 
who have developed an addiction after LT. To test the hypothesis that both decreases in alcohol use disorders and increases in food addiction are causally related, we will regularly (once a year) assess both disorders using appropriate and reliable measures: food addiction will be assessed using the Yale Food Addiction Scale, the gold standard for such assessments [15,63]; alcohol addiction will be assessed using semi-structured interviews based on DSM-5 criteria. Because it is often difficult for patients to tell the transplantation team that they have relapsed [64] and because medical record reviews alone greatly underestimate alcohol relapse rates [6], we will establish whether relapse indeed occurred using repeated measurements with complementary methods, including a patient self-report, a collateral report, and biological measures [6,65]. We will also separate the study staff from the transplantation staff to keep notifications of alcohol or drug use confidential within the study [64].

To demonstrate that the increased prevalence of obesity, weight gain and metabolic syndrome after LT could be causally related to food addiction, we will assess not only body mass index but also the prevalence of diabetes, dyslipidemia and hypertension. If this association is confirmed, we will then search for explanations that might account for this significant association. To demonstrate that this switch occurs in a vulnerable subpopulation, we will jointly investigate the neurobiological, psychiatric and psychological factors that are typically associated with both food addiction and alcohol addiction, at both the clinical and preclinical levels. These factors include personality dimensions (which we will measure using the Big Five Inventory, a brief and reliable measure of the five common personality dimensions) [66,67], personality traits (such as impulsivity, which can be measured for example using the Barratt Impulsiveness Scale) [68] and psychiatric disorders (attention deficit hyperactivity disorder, major depression, anxiety disorders, personality disorders, which can be measured using clinical interviews based on DSM criteria) [1].

However, in human studies, for obvious ethical reasons, it is difficult to disentangle LT's contribution to metabolic regulation from the processes that depend on the pharmacological treatments that are associated with LT, namely, immunosuppressive drugs such as cyclosporine. Indeed, the latter have been suggested to potentially contribute to post-LT obesity, MS and lower rates of alcohol relapse $[8,14,69]$. We will therefore use preclinical models in rats with great heuristic value with regard to habitual and compulsive alcohol [70] and food seeking and taking [71] to identify the neurobiological substrates of the effect of cyclosporine treatment on relapse to alcohol or food-seeking behaviour and the potential switch from alcohol. Thus, we propose to address these issues in a preclinical model of habitual and compulsive alcohol and food seeking. Animal studies will also be helpful in studying the polymorphisms in orexin genes that could contribute to alcohol relapse or food addiction after LT.

Finally, we will also implement longitudinal studies in rats that aim at identifying whether specific behavioural markers such as novelty seeking, impulsivity, decision making, sweetness preference, sign tracking, behavioural flexibility and anxiety [61,72-75] contribute to an increased propensity to switch from alcohol to compulsive food seeking with or without cyclosporine treatment following self-abstinence triggered by repeated adverse consequences related to alcohol seeking [76].

This combination of a clinical and preclinical model will provide the scientific community with a new post-LT prognostic approach, thereby unlocking both a scientific and technical barrier: better insight into the psychological and biological dimensions that are associated with post-transplant food addiction, metabolic syndrome and obesity and a preclinical model of the switch from a previous addiction to food addiction. The model has clear medical and translational implications because it should provide new avenues for treating the primary post-LT impairments, namely obesity, metabolic syndrome, food addiction and alcohol addiction.

\section{CONSEQUENCES OF THE HYPOTHESIS: DISCUSSION}

Provided that it is confirmed, the present hypothesis may yield to a novel treatment strategy for helping some patients benefit from LT, as well as to new avenues for future research not only in liver-transplanted patients but also in patients who are followed up for alcohol addiction.

Implications for liver-transplanted patients include the fact that patients with a history of pre-transplant alcohol addiction should be systematically screened after LT for food addiction and followed up for subsequent risk of obesity and metabolic syndrome. These patients should also be screened for psychiatric disorders both pre 
and post LT. A subpopulation of vulnerable individuals who develop post-LT obesity or metabolic syndrome could also be referred to addiction units in order to screen for and treat food addiction. Interventions that target risk factors for both alcohol and food addiction in the pre- and post-transplant periods could also reduce the prevalence of these two disorders after LT. Such interventions are critical because they could not only improve survival, comorbidities and health-related quality of life after LT but also help to address the critical shortage of donor organs and increase public willingness to donate.

Such an integrative assessment would enable the early recognition of food addiction and alcohol addiction and their risk factors, as well as early screening for psychiatric comorbidities that could potentially diminish quality of life if left untreated. These studies could help to build a simple and practical tool for clinicians that could include assessing the specific pre- and post-LT psychiatric and personality dimensions that are associated with post-LT food and alcohol addiction and poorer outcomes. This assessment is also a preliminary step before effective psychopharmacological and psychotherapeutic strategies that target these specific risk factors can be tested. Given that the drugs used to treat both of these disorders are primarily metabolised by the liver, studies that focus on the efficacy and side effects of psychopharmacological strategies should take into account the prominent concern about safety and overdosing in this population.

Finally, the dimension of insight should be systematically assessed after LT so that psychotherapeutic interventions that target insight itself or the psychopathological factors associated with it might be undertaken. If low insight is confirmed to be a risk factor for subsequent alcohol addiction, psychotherapeutic strategies such as motivational interviewing could be effective and should be tested in these populations. Such an approach finally aims to improve patients' survival, outcomes and quality of life following LT.

Our addiction transfer model could also be tested and generalised for patients in the overall population who are treated for alcohol addiction because current studies suggest that patients who stop alcohol use are at higher risk of developing food addiction, thereby leading to an increased risk of obesity and metabolic syndrome. Investigation of our model might also help discover neurobiological, psychiatric and psychological risk factors for both alcohol addiction and food addiction. These studies would help us improve our understanding of the links between alcohol addiction, food addiction and obesity and, ultimately, to developing more efficient therapeutic strategies for these patients. Future studies should also determine whether our addiction transfer model could also be extrapolated to other patients; it could, for example, explain why some patients who are treated for food addiction develop alcohol addiction after treatment (i.e., in a subpopulation of bariatric surgery patients), why patients who are treated for alcohol addiction may be at higher risk of switching, and this model could even explain addiction transfers between alcohol and other addictions (i.e., other drug and behavioural addictions).

\section{Conflicts of interest statement}

DB is supported by the University of Cambridge and the Agence Nationale de la Recherche (ANR) grant ANR12 SAMA00201.

PB has received financial support from Lundbeck to participate in two sessions of continuing medical education at the Lundbeck Institute (2012 and 2013) and to attend three medical meetings (2013 and 2014); from DNA Pharma in 2014 for participating in a clinical trial as an associated investigator; and from Astra Zeneca to attend two medical meetings (2014).

NB received financial support as chairman for medical meetings from Astra Zeneca in 2010 and Lundbeck in 2013 and 2014 and as co-investigator in a study sponsored by DNA Pharma in 2012-2013.

\section{References}

[1] American Psychiatric Association. Diagnostic and Statistical Manual of mental disorders (5th ed.). Arlington, VA: American Psychiatric Publishing, Inc.; 2013.

[2] Merikangas KR, McClair VL. Epidemiology of substance use disorders. Hum Genet 2012;131:779-89. doi:10.1007/s00439012-1168-0.

[3] Rehm J, Mathers C, Popova S, Thavorncharoensap M, Teerawattananon Y, Patra J. Global burden of disease and injury and economic cost attributable to alcohol use and alcohol-use disorders. Lancet 2009;373:2223-33. doi:10.1016/S0140-6736(09)60746-7. 
[4] Guérin S, Laplanche A, Dunant A, Hill C. Alcohol-attributable mortality in France. Eur J Public Health 2013;23:588-93. doi:10.1093/eurpub/ckt015.

[5] Matesanz R, Coll E, Dominguez-Gil B, Mahillo B, Escobar EM, Garrido G. Global Education Initiatives. Transplantation 2009;88:S43-6.

[6] Dew MA, DiMartini AF, Steel J, De Vito Dabbs A, Myaskovsky L, Unruh M, et al. Meta-analysis of risk for relapse to substance use after transplantation of the liver or other solid organs. Liver Transpl 2008;14:159-72. doi:10.1002/1t.21278.

[7] Iruzubieta P, Crespo J, Fábrega E. Long-term survival after liver transplantation for alcoholic liver disease. World J Gastroenterol 2013;19:9198-208. doi:10.3748/wjg.v19.i48.9198.

[8] Pagadala M, Dasarathy S, Eghtesad B, McCullough AJ. Posttransplant metabolic syndrome: an epidemic waiting to happen. Liver Transpl 2009;15:1662-70. doi:10.1002/lt.21952.

[9] Anastácio LR, Lima AS, Toulson Davisson Correia MI. Metabolic syndrome and its components after liver transplantation: incidence, prevalence, risk factors, and implications. Clin Nutr 2010;29:175-9. doi:10.1016/j.clnu.2009.08.008.

[10] Anastácio LR, Ferreira LG, de Sena Ribeiro H, Liboredo JC, Lima AS, Toulson Davisson Correia MI. Metabolic syndrome after liver transplantation: prevalence and predictive factors. Nutrition 2011;27:931-7. doi:10.1016/j.nut.2010.12.017.

[11] Yeomans MR. Alcohol, appetite and energy balance: is alcohol intake a risk factor for obesity? Physiol Behav 2010;100:82-9. doi:10.1016/j.physbeh.2010.01.012.

[12] Beresford TP. Probabilities of relapse and abstinence among liver transplant recipients. Liver Transpl 2006;12:705-6. doi:10.1002/lt.20752.

[13] Malet L, Reynaud M, Llorca P-M, Chakroun N, Blanc O, Falissard B. Outcomes from primary care management of alcohol dependence in France. J Subst Abuse Treat 2009;36:457-62. doi:10.1016/j.jsat.2008.08.006.

[14] Correia MI, Rego LO, Lima AS. Post-liver transplant obesity and diabetes. Curr Opin Clin Nutr Metab Care 2003;6:457-60. doi:10.1097/01.mco.0000078994.96795.d8.

[15] Brunault P, Ballon N, Gaillard P, Réveillère C, Courtois R. Validation of the French version of the Yale Food Addiction Scale: an examination of its factor structure, reliability, and construct validity in a nonclinical sample. Can J Psychiatry 2014;59:276-84. doi:PMID:25007281; PMCID: PMC4079141.

[16] Gearhardt AN, Corbin WR, Brownell KD. Food addiction: an examination of the diagnostic criteria for dependence. J Addict Med 2009;3:1-7.

[17] Ifland JR, Preuss HG, Marcus MT, Rourke KM, Taylor WC, Burau K, et al. Refined food addiction: a classic substance use disorder. Med Hypotheses 2009;72:518-26. doi:10.1016/j.mehy.2008.11.035.

[18] Gearhardt AN, White MA, Masheb RM, Grilo CM. An examination of food addiction in a racially diverse sample of obese patients with binge eating disorder in primary care settings. Compr Psychiatry 2013;54:500-5. doi:10.1016/j.comppsych.2012.12.009.

[19] Meule A. Food addiction and body-mass-index: A non-linear relationship. Med Hypotheses 2012;79:508-11. doi:10.1016/j.mehy.2012.07.005.

[20] Corsica JA, Pelchat ML. Food addiction: true or false? Curr Opin Gastroenterol 2010;26:165-9. doi:10.1097/MOG.0b013e328336528d.

[21] King WC, Chen JY, Mitchell JE, Kalarchian MA, Steffen KJ, Engel SG, et al. Prevalence of alcohol use disorders before and after bariatric surgery. JAMA 2012;307:2516-25. doi:10.1001/jama.2012.6147.

[22] Blum K, Bailey J, Gonzalez AM, Oscar-Berman M, Liu Y, Giordano J, et al. Neuro-Genetics of Reward Deficiency Syndrome (RDS) as the Root Cause of "Addiction Transfer": A New Phenomenon Common after Bariatric Surgery. J Genet Syndr Gene Ther 2011;2012. doi:10.4172/2157-7412.S2-001.

[23] Gearhardt AN, Yokum S, Orr PT, Stice E, Corbin WR, Brownell KD. Neural correlates of food addiction. Arch Gen Psychiatry 2011;68:808-16. doi:10.1001/archgenpsychiatry.2011.32.

[24] Von Deneen KM, Liu Y. Food Addiction, Obesity and Neuroimaging (pp. 259-290). In: Belin D, editor. Addictions - From pathophysiology to treatment. InTech; 2012. Available at: http://www.intechopen.com/books/addictions-from-pathophysiology-to-treatment [25] Corwin RL, Avena NM, Boggiano MM. Feeding and reward: perspectives from three rat models of binge eating. Physiol Behav 2011;104:87-97. doi:10.1016/j.physbeh.2011.04.041.

[26] Belin D, Jonkman S, Dickinson A, Robbins TW, Everitt BJ. Parallel and interactive learning processes within the basal ganglia: relevance for the understanding of addiction. Behav Brain Res 2009;199:89-102. doi:10.1016/j.bbr.2008.09.027.

[27] Smith DG, Robbins TW. The Neurobiological Underpinnings of Obesity and Binge Eating: A Rationale for Adopting the Food Addiction Model. Biol Psychiatry 2013;73:804-10. doi:10.1016/j.biopsych.2012.08.026.

[28] Volkow ND, Wang GJ, Fowler JS, Tomasi D, Baler R. Food and drug reward: overlapping circuits in human obesity and addiction. Curr Top Behav Neurosci 2012;11:1-24.doi:10.1007/7854_2011_169.

[29] Volkow ND, Wang G-J, Tomasi D, Baler RD. The addictive dimensionality of obesity. Biol Psychiatry 2013;73:811-8. doi:10.1016/j.biopsych.2012.12.020.

[30] Fortuna JL. Sweet preference, sugar addiction and the familial history of alcohol dependence: shared neural pathways and genes. J Psychoactive Drugs 2010;42:147-51. doi:PMID: 20648910. 
[31] Krahn D, Grossman J, Henk H, Mussey M, Crosby R, Gosnell B. Sweet intake, sweet-liking, urges to eat, and weight change: relationship to alcohol dependence and abstinence. Addict Behav 2006;31:622-31. doi:10.1016/j.addbeh.2005.05.056.

[32] Hodgkins C, Frost-Pineda K, Gold MS. Weight gain during substance abuse treatment. J Addict Dis 2007;26:41-50. doi:10.1300/J069v26S01_05.

[33] Hodgkins CC, Cahill KS, Seraphine AE, Frostpineda K, Gold MS. Adolescent drug addiction treatment and weight Gain. J Addict Dis 2004;23:55-65. doi:10.1300/J069v23n03_05.

[34] Walfish S, Stenmark DE, Sarco D, Shealy JS, Krone AM. Incidence of bulimia in substance misusing women in residential treatment. Subst Use Misuse 1992;27:425-33.

[35] Tanofsky-Kraff M, Bulik CM, Marcus MD, Striegel RH, Wilfley DE, Wonderlich SA, et al. Binge eating disorder: The next generation of research. Int J Eat Disord 2013;46:193-207. doi:10.1002/eat.22089.

[36] Sonneville KR, Horton NJ, Micali N, Crosby RD, Swanson SA, Solmi F, et al. Longitudinal associations between binge eating and overeating and adverse outcomes among adolescents and young adults: Does loss of control matter? JAMA Pediatr 2013;167:149-55. doi:10.1001/2013.jamapediatrics.12.

[37] Loos RJF, Bouchard C. Obesity - is it a genetic disorder? J Intern Med 2003;254:401-25. doi:10.1046/j.13652796.2003.01242.x.

[38] Farley AC, Hajek P, Lycett D, Aveyard P. Interventions for preventing weight gain after smoking cessation. Cochrane Database Syst Rev 2012;1:CD006219. doi:10.1002/14651858.CD006219.pub3.

[39] Kahl KG, Greggersen W, Schweiger U, Cordes J, Correll CU, Ristow J, et al. Prevalence of the metabolic syndrome in men and women with alcohol dependence: results from a cross-sectional study during behavioural treatment in a controlled environment. Addiction 2010;105:1921-7. doi:10.1111/j.1360-0443.2010.03059.x.

[40] Kelly NR, Bulik CM, Mazzeo SE. Executive functioning and behavioral impulsivity of young women who binge eat. Int J Eat Disord 2013;46:127-39. doi:10.1002/eat.22096.

[41] Van Holst RJ, Schilt T. Drug-related decrease in neuropsychological functions of abstinent drug users. Curr Drug Abuse Rev 2011;4:42-56. doi:PMID: 21466500.

[42] Conway KP, Compton W, Stinson FS, Grant BF. Lifetime comorbidity of DSM-IV mood and anxiety disorders and specific drug use disorders: results from the National Epidemiologic Survey on Alcohol and Related Conditions. J Clin Psychiatry 2006;67:247-57. doi:PMID: 16566620.

[43] Gearhardt AN, White MA, Masheb RM, Morgan PT, Crosby RD, Grilo CM. An examination of the food addiction construct in obese patients with binge eating disorder. Int J Eat Disord 2012;45:657-63. doi:10.1002/eat.20957.

[44] Cortese S, Bernardina BD, Mouren M-C. Attention-deficithyperactivity disorder (ADHD) and binge eating. Nutr Rev 2007;65:404-11. doi:PMID: 17958207.

[45] Wilens TE, Morrison NR. The intersection of attention-deficit/hyperactivity disorder and substance abuse. Curr Opin Psychiatry 2011;24:280-5. doi:10.1097/YCO.0b013e328345c956.

[46] Ferriter C, Ray LA. Binge eating and binge drinking: an integrative review. Eat Behav 2011;12:99-107. doi:10.1016/j.eatbeh.2011.01.001.

[47] Meule A, Lutz A, Vögele C, Kübler A. Women with elevated food addiction symptoms show accelerated reactions, but no impaired inhibitory control, in response to pictures of high-calorie food-cues. Eat Behav 2012;13:423-8. doi:10.1016/j.eatbeh.2012.08.001.

[48] Volkow ND, Wang G-J, Fowler JS, Telang F. Overlapping neuronal circuits in addiction and obesity: evidence of systems pathology. Philos Trans R Soc B Biol Sci 2008;363:3191-200.

[49] Volkow ND, Wise RA. How can drug addiction help us understand obesity? Nat Neurosci 2005;8:555-60. doi:10.1038/nn1452. [50] Brown RM, Lawrence AJ. Ascending orexinergic pathways and alcohol-seeking. Curr Opin Neurobiol 2013;23:467-72. doi:10.1016/j.conb.2013.02.014.

[51] Cason AM, Smith RJ, Tahsili-Fahadan P, Moorman DE, Sartor GC, Aston-Jones G. Role of orexin/hypocretin in rewardseeking and addiction: implications for obesity. Physiol Behav 2010;100:419-28. doi:10.1016/j.physbeh.2010.03.009.

[52] Chen Y-W, Barson JR, Chen A, Hoebel BG, Leibowitz SF. Glutamatergic input to the lateral hypothalamus stimulates ethanol intake: role of orexin and melanin-concentrating hormone. Alcohol Clin Exp Res 2013;37:123-31. doi:10.1111/j.1530-0277.2012.01854.x. [53] Di Sebastiano AR, Coolen LM. Orexin and natural reward: feeding, maternal, and male sexual behavior. Prog Brain Res 2012;198:65-77. doi:10.1016/B978-0-444-59489-1.00006-9.

[54] Sakurai T, Amemiya A, Ishii M, Matsuzaki I, Chemelli RM, Tanaka H, et al. Orexins and orexin receptors: a family of hypothalamic neuropeptides and G Protein-coupled receptors that regulate feeding behavior. Cell 1998;92:573-85. doi:10.1016/S00928674(00)80949-6.

[55] Bossert JM, Ghitza UE, Lu L, Epstein DH, Shaham Y. Neurobiology of relapse to heroin and cocaine seeking: an update and clinical implications. Eur J Pharmacol 2005;526:36-50. doi:PMID: 16289451.

[56] Shalev U, Erb S, Shaham Y. Role of CRF and other neuropeptides in stress-induced reinstatement of drug seeking. Brain Res 2010;1314:15-28. doi:10.1016/j.brainres.2009.07.028.

[57] Tomasi D, Volkow ND. Striatocortical pathway dysfunction in addiction and obesity: differences and similarities. Crit Rev Biochem Mol Biol 2013;48:1-19. doi:10.3109/10409238.2012.735642.

[58] Marková I. Insight in psychiatry. Cambridge: Cambridge University Press; 2005. 
[59] Paillot C, Ingrand P, Millet B, Amador XF, Senon JL, Olié JP, et al. [French translation and validation of the Scale to assess Unawareness of Mental Disorder (SUMD) in patients with schizophrenics]. Encéphale 2010;36:472-7. doi:10.1016/j.encep.2009.12.008.

[60] Lacoste J, Daniel M-L, Meissonnier F, Bacconnier M, Senon J-L, Belin D, et al. [Insight and motivational interviewing effectiveness on relapse prevention in alcohol dependent patients]. Ann Med Psychol Paris 2011;169:457-8.

[61] Belin D, Mar AC, Dalley JW, Robbins TW, Everitt BJ. High impulsivity predicts the switch to compulsive cocaine-taking. Science 2008;320:1352-5. doi:10.1126/science.1158136.

[62] Belin-Rauscent A, Everitt BJ, Belin D. Intrastriatal shifts mediate the transition from drug-seeking actions to habits. Biol Psychiatry 2012;72:343-5. doi:10.1016/j.biopsych.2012.07.001.

[63] Gearhardt AN, Corbin WR, Brownell KD. Preliminary validation of the Yale Food Addiction Scale. Appetite 2009;52:430-6. doi:10.1016/j.appet.2008.12.003.

[64] Tome S, Said A, Lucey MR. Addictive behavior after solid organ transplantation: what do we know already and what do we need to know? Liver Transpl 2008;14:127-9. doi:10.1002/lt.21311.

[65] McCallum S, Masterton G. Liver transplantation for alcoholic liver disease: a systematic review of psychosocial selection criteria. Alcohol Alcohol 2006;41:358-63. doi:10.1093/alcalc/agl033.

[66] John OP, Donahue EM, Kentle RL. The Big Five Inventory--Versions 4a and 54. Berkeley, CA: University of California, Berkeley, Institute of Personality and Social Research; 1991.

[67] Plaisant O, Courtois R, Réveillère C, Mendelsohn G, John O. [Factor structure and internal reliability of the French Big Five Inventory (BFI-Fr). Convergent and discriminant validation with the NEO-PI-R]. Ann Med Psychol Paris 2010;168:97-106. doi:10.1016/j.amp.2009.09.003.

[68] Stanford MS, Mathias CW, Dougherty DM, Lake SL, Anderson NE, Patton JH. Fifty years of the Barratt Impulsiveness Scale: An update and review. Personal Individ Differ 2009;47:385-95.

[69] Beresford HF, Deitrich R, Beresford TP. Cyclosporine-A discourages ethanol intake in C57bl/6j mice: a preliminary study. J Stud Alcohol 2005;66:658-62. doi:PMID: 16331851.

[70] Vanhille N, Ducret E, Puaud M, Ansquer S, Fouyssac M, Houeto J-L, et al. C.20 - Differential effect of environmental enrichment on the acquisition of drug use and the transition to addiction. Behav Pharmacol 2013;24:e35-6. doi:10.1097/01.fbp.0000434804.04205.49.

[71] Giuliano C, Robbins TW, Nathan PJ, Bullmore ET, Everitt BJ. Inhibition of opioid transmission at the $\mu$-opioid receptor prevents both food seeking and binge-like eating. Neuropsychopharmacology 2012;37:2643-52.

[72] Vanhille N, Belin-Rauscent A, Mar AC, Ducret E, Belin D. High Locomotor Reactivity to Novelty Is Associated with an Increased Propensity to Choose Saccharin Over Cocaine: New Insights into the Vulnerability to Addiction. Neuropsychopharmacology 2015;40:577-89.

[73] Murray JE, Dilleen R, Pelloux Y, Economidou D, Dalley JW, Belin D, et al. Increased impulsivity retards the transition to dorsolateral striatal dopamine control of cocaine seeking. Biol Psychiatry 2014;76:15-22. doi:10.1016/j.biopsych.2013.09.011. [74] Dilleen R, Pelloux Y, Mar AC, Molander A, Robbins TW, Everitt BJ, et al. High anxiety is a predisposing endophenotype for loss of control over cocaine, but not heroin, self-administration in rats. Psychopharmacology (Berl) 2012;222:89-97. doi:10.1007/s00213011-2626-4.

[75] Molander AC, Mar A, Norbury A, Steventon S, Moreno M, Caprioli D, et al. High impulsivity predicting vulnerability to cocaine addiction in rats: some relationship with novelty preference but not novelty reactivity, anxiety or stress. Psychopharmacology (Berl) 2011;215:721-31. doi:10.1007/s00213-011-2167-x.

[76] Marchant NJ, Khuc TN, Pickens CL, Bonci A, Shaham Y. Context-induced relapse to alcohol seeking after punishment in a rat model. Biol Psychiatry 2013;73:256-62. doi:10.1016/j.biopsych.2012.07.007. 
Figure 1. In patients who either relapse to alcohol or develop food addiction after LT, low insight predicts an increased propensity to relapse to alcohol addiction, whereas high insight relapse predicts an increased propensity to develop food addiction.

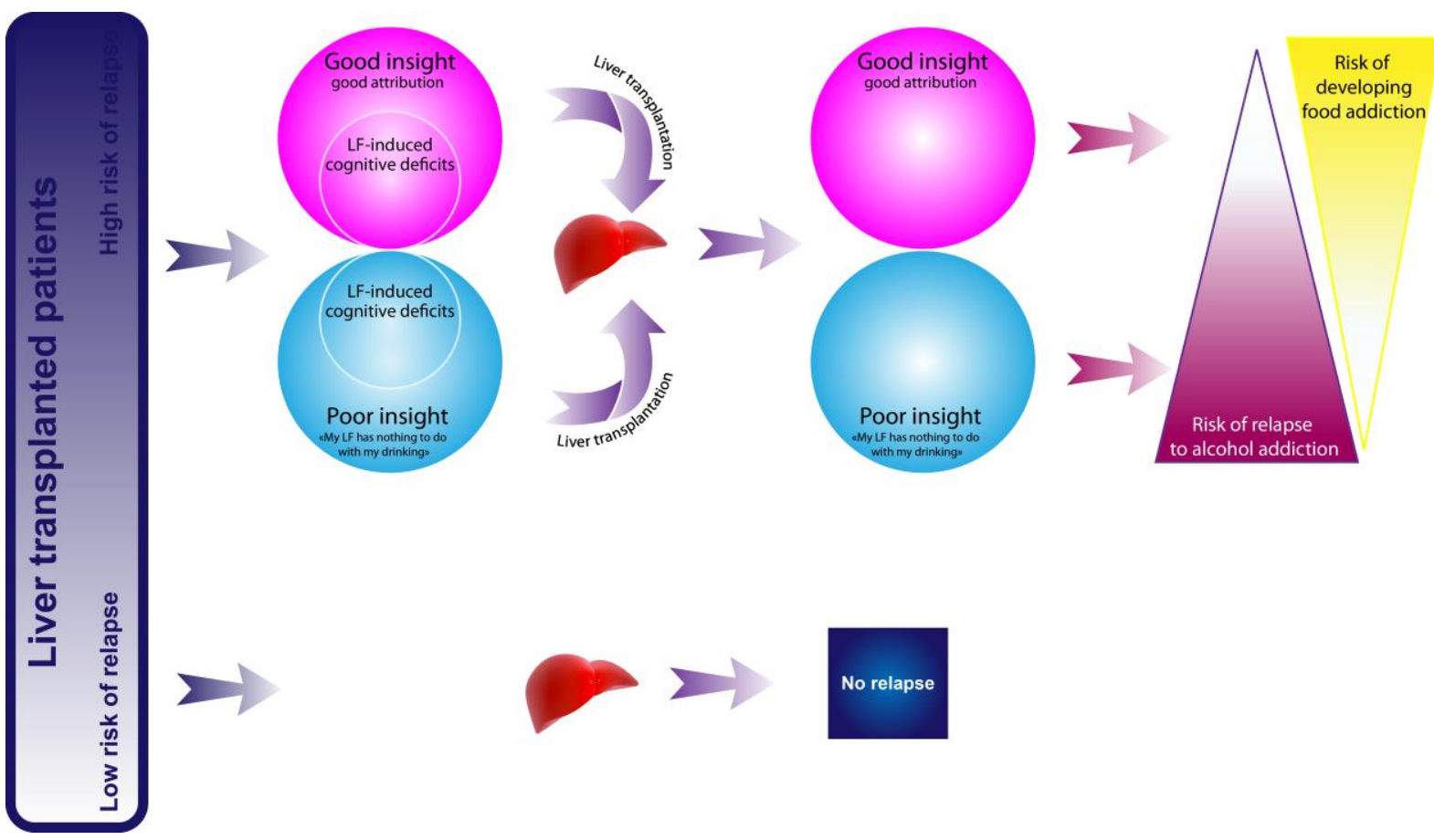

FIGURE LEGEND:

This figure differentiates between (1) patients who relapse to alcohol or develop food addiction after LT (top of the figure) and (2) patients who do not (bottom of the figure). As illustrated in the left part of the figure, the first population is more likely to have a high risk of relapse (as assessed by the combination of psychobiological factors that we have previously discussed in the paper), whereas the second population is more likely to have a low risk of relapse (as assessed by the same combination of psychobiological factors).

In patients who develop addiction after LT (top of the figure), we hypothesise that the level of insight with regard to the somatic and psychological consequences of alcohol might predict either alcohol relapse or the development of food addiction after LT. In this population and after LT, we hypothesise that patients with low insight (e.g., "my liver failure has nothing to do with my drinking behaviour") are at higher risk for relapsing to alcohol addiction, whereas patients with high insight (e.g., "I recognise that my drinking behaviour is responsible for my liver failure") are at higher risk for developing food addiction. Before the diagnosis of liver failure (LF) and LT, these patients might have experienced a different type of insight that was more difficult to assess because it was affected by LF-induced cognitive deficits.

LF: Liver Failure.

LT: Liver Transplantation. 